\title{
Gender-specific regulation of mitochondrial fusion and fission gene transcription and viability of cortical astrocytes by steroid hormones
}

\author{
Susanne Arnold, Gilda Wright de Araújo and Cordian Beyer \\ Faculty of Medicine, Institute for Neuroanatomy, RWTH Aachen University, 52074 Aachen, Germany \\ (Correspondence should be addressed to S Arnold; Email: sarnold@ukaachen.de)
}

\begin{abstract}
Astroglia and steroid hormones such as estrogen and progesterone regulate cell growth, function, and protection in the central nervous system (CNS). It appears that astrocytes and steroids act in concert to promote cell survival under pathological conditions. With respect to the role of mitochondrial fusion and fission in energy metabolism, apoptosis, and proliferation, astrocyte mitochondria resemble a perfect intracellular target for steroids to modulate these processes, thereby promoting cell vitality after damage. We have studied the effects of estrogen and progesterone on cell viability in comparison with mitochondrial fusion and fission gene transcription in primary cortical astrocytes from female and male mouse brains. Estrogen- and progesterone-treated female astrocytes demonstrated an increase in cell number and proliferation marker accompanied by an upregulation of fusion and fission gene transcription, which were apparently balancing pro- and anti-apoptotic processes. On the other hand, male astrocytes exhibited no change in cell number after estrogen treatment, but a decrease after progesterone administration. This could be the consequence of stimulated apoptosis in male astrocytes by both steroids, which was counterbalanced by an increased proliferation in the presence of estrogen, whereas it was strengthened in the presence of progesterone. Supportively, estrogen promoted and progesterone decreased the transcription of fusion and fission genes. We suggest that estrogen and progesterone affect mitochondrial fusion and fission gene transcription in cortical astrocytes in a gender-specific way, thereby influencing mitochondrial function differently in both genders. Thus, interaction of sex steroids with mitochondria may represent one possible cause for gender differences in cellular pathology in the CNS.
\end{abstract}

Journal of Molecular Endocrinology (2008) 41, 289-300

\section{Introduction}

Estrogen (E) and progesterone (P) exert their wellknown neuroprotective effects in the central nervous system (CNS) by initiating a multitude of cellular mechanisms that are often mediated by astrocytes (Azcoitia et al. 2001, Arnold 2005, Pawlak et al. 2005, Garcia-Segura \& Melcangi 2006, Leonelli et al. 2007). Astrocyte mitochondria represent an interesting intracellular target organelle for steroid hormones, since they provide ample of services to neural cells including energy production, calcium buffering, and regulation of apoptosis. These processes are also known to be modulated by gonadal steroid hormones (Nilsen \& Brinton 2004, Kipp et al. 2006). However, mitochondriamediated mechanisms in steroid-dependent protection are not fully understood. In very recent studies, we and others have shown that $\mathrm{E}$ promotes the expression of mitochondria-encoded subunits of the respiratory chain, thereby influencing mitochondrial respiratory activity and enhancing functional efficiency (Araújo et al. 2008, Irwin et al. 2008). Mitochondrial functions are not only affected by regulation of gene expression and activity of the respiratory chain (Zheng \& Ramirez 1999, Araújo et al. 2008, Irwin et al. 2008), but also by the morphology of these organelles. Two sets of proteins and their relative activities are involved in shaping the morphology of mitochondria. While mitochondrial GTPases, mitofusin 1 and 2 (MFN1 and MFN2), and optic atrophy 1 homolog (OPA1), are essential for fusion processes, fission 1 (mitochondrial outer membrane) homolog (FIS1) and dynamin-related protein 1 (DNM1L) are essential fission proteins (Santel \& Fuller 2001, Rojo et al. 2002, Chen et al. 2003, Olichon et al. 2003, Cipolat et al. 2004, Griparic et al. 2004, Chen \& Chan 2005). Given the highly ordered arrangement of mitochondrial networks in some cell types (Bakeeva et al. 1981), it is likely that mitochondrial shape indeed has important consequences for mitochondrial function. Under physiological conditions, mitochondria are characterized by a dynamic equilibrium of fusion and fission, whereas an imbalanced expression of fusion versus fission proteins leads to a disturbance of this equilibrium and causes dramatic changes in mitochondrial morphology and function (Yaffe 1999, Karbowski \& Youle 2003). In humans, mutations in 
Mfn2 cause Charcot-Marie-Tooth neuropathy type 2A (Zuchner et al. 2004, Kijima et al. 2005). Mutations in Opal lead to autosomal dominant optic atrophy (Alexander et al. 2000, Delettre et al. 2000). A downregulation of fusion proteins together with an impairment of mitochondrial fusion processes or an overexpression of fission proteins causes fragmentation of the tubular mitochondrial network (James et al. 2003, Olichon et al. 2003, Yoon et al. 2003, Lee et al. 2004, Stojanovski et al. 2004, Chen et al. 2005, Detmer \& Chan $2007 a, b)$. As a consequence, an increased susceptibility to cell death and apoptosis occurred. Knock-out mice of either $M f n 1$ or $M f n 2$ are both embryonic lethal demonstrating the essential role of mitochondrial fusion for embryonal development and cell viability (Chen et al. 2003). In contrast, overexpression of the two MFN proteins together provides some protection against different apoptotic stimuli (Sugioka et al. 2004). On the other hand, mitochondrial fission proteins (FIS1 and DNM1L) and processes are essential for cell division as well as for programmed cell death and decreased cell survivability (James et al. 2003, Yoon et al. 2003, Lee et al. 2004, Stojanovski et al. 2004). In Caenorhabditis elegans, an overexpression of fission protein DNM1L increased the number of mitochondrial divisions (Labrousse et al. 1999). FIS1 overexpression also caused mitochondrial fragmentation and aggregation (Stojanovski et al. 2004). On the contrary, a disruption of fission by overexpressing a mutant form of DNM1L or overexpression of fusion proteins provoked an elongation and formation of interconnected mitochondrial network due to enhanced mitochondria fusion (Smirnova et al. 2001, Santel et al. 2003).

Considering the severe consequences of imbalanced mitochondrial fusion and fission for neurodegeneration, we studied the effect of $\mathrm{E}$ and $\mathrm{P}$ on the transcription level of mitochondrial fusion and fission genes in cortical astrocytes in vitro. Astrocytes were chosen since they are the outstanding cell type in the CNS supporting neuronal viability and function. It is apparent that neurodegenerative processes and their steroid responsiveness often occur in a gender-specific way (Miller et al. 1998, Roof \& Hall 2000, van den Eeden et al. 2003, Dluzen \& McDermott 2004, Kenchappa et al. 2004, Baba et al. 2005, Marchetti et al. 2005). Therefore, we have analyzed female and male astroglia separately.

\section{Materials and methods}

\section{Materials}

All chemicals were obtained from Roth (Karlsruhe, Germany), whereas reagents for molecular biological techniques and cell culture were purchased from Invitrogen, unless indicated otherwise.

\section{Animals}

BALB/c mice were purchased from Harlan Winkelmann GmbH (Borchen, Germany). All procedures were performed in strict accordance with the published welfare rules for the care and use of laboratory animals at the University Clinic Aachen and the government of the State of Nordrhein-Westfalen, Germany.

\section{Preparation of primary astrocytes}

Astrocytes were prepared from postnatal day 1 BALB/c mice (Pawlak et al. 2005, Horvat et al. 2006). The gender of the donor was determined by visual inspection of the anogenital distance that is larger in males (Beyer et al. 1991). Brains from decapitated mice were removed and transferred in preparation buffer consisting of $10 \mathrm{mM}$ HEPES, $154 \mathrm{mM} \mathrm{NaCl}, 10 \mathrm{mM}$ glucose, $2 \mathrm{mM} \mathrm{KCl}$, and $15 \mu \mathrm{M}$ BSA. The brain cortices were separated and meninges removed. Afterwards, the brain tissue was incubated in PBS containing $0 \cdot 1 \%(\mathrm{w} / \mathrm{v})$ trypsin and $0.02 \%(\mathrm{w} / \mathrm{v})$ EDTA for $15 \mathrm{~min}$ and minced with a Pasteur pipette and filtered through a $50 \mu \mathrm{m}$ nylon mesh. The cell suspension was centrifuged at $300 \mathrm{~g}$ (Eppendorf, Hamburg, Germany) for $4 \mathrm{~min}$. The cell pellet was re-suspended and the cell suspension plated onto poly-L-ornithine-coated (Sigma-Aldrich) culture dishes and cultured in Dulbecco's modified Eagle's medium (PAA, Coelbe, Germany) supplemented with $20 \%(\mathrm{v} / \mathrm{v})$ fetal calf serum (PAA), $50 \mathrm{U} / \mathrm{ml}$ penicillin, $50 \mu \mathrm{g} / \mathrm{ml}$ streptomycin, $0 \cdot 25 \mu \mathrm{g} / \mathrm{ml}$ amphotericin B (Fungizone), and $2 \mathrm{mM}$ glutamine (Glutamax). Cultures were maintained at $37^{\circ} \mathrm{C}$ in a humidified atmosphere of $95 \%$ air $/ 5 \% \mathrm{CO}_{2}$. Before reaching confluence, astrocytes were trypsinized and plated at lower density. Subconfluent cells were incubated in neurobasal medium (NBM) supplemented with $0 \cdot 2 \%(\mathrm{v} / \mathrm{v}) \mathrm{B} 27$, penicillin, streptomycin, Fungizone, and L-glutamine for $48 \mathrm{~h}$. Astrocytes were subsequently used for experiments.

\section{Cell treatment}

Cortical astrocytes were placed on culture dishes in a humidified atmosphere at $37^{\circ} \mathrm{C}$ in the presence of NBM and were treated with $10^{-7} \mathrm{M} 17 \beta$-estradiol (E) or $\mathrm{P}$ for $24 \mathrm{~h}$. To inhibit nuclear estrogen receptors (ER) and progesterone receptors (PR), cells were simultaneously treated with $10^{-6}$ M ICI 182780 (ICI; Tocris, Bristol, UK) and $10^{-6}$ M Rti 3021-022 (Rti; SigmaAldrich) respectively. Cells of the same preparation maintained under identical conditions except for hormone and antagonist treatments served as controls.

\section{Cell viability}

Primary astrocytes from brain cortices were treated as described above and mentioned in the figure legends. 
In brief, cells were plated on cover slips, treated, and stained with $1 \mu \mathrm{g} / \mathrm{ml}$ Hoechst 33342 Trihydrochlorid (Hoechst, Invitrogen) for 5 min under culturing conditions. Subsequently, cells were washed twice with PBS, fixed with $4 \%(\mathrm{w} / \mathrm{v})$ paraformaldehyde (Merck) for $15 \mathrm{~min}$ at room temperature, washed twice with PBS, and mounted in Mowiol (Merck). Hoechst-stained normal, apoptotic, and necrotic cell nuclei were detected with a Hitachi HV-C20A Camera connected to a fluorescence microscope (Leica Microsystem, Wetzlar, Germany) at excitation wavelength of $365 \mathrm{~nm}$. Cell nuclei were scored accordingly to their specific morphological characteristics (Horvat et al. 2006) from the images taken by using the Adobe Photoshop CS2 software (Adobe Systems Inc). Viable and apoptotic cells were counted from five distinct areas of the same size per cover slip from three to five independent experiments. Cell numbers were calculated as percentage of the ratio of sample cell number to control cell number referred to as $100 \%$. Additionally, cells were stained with propidium iodide to visualize necrotic astrocytes. Before the cells were stained with $500 \mathrm{nM}$ propidium iodide for $10 \mathrm{~min}$, they were fixed in $100 \%$ (v/v) ice-cold methanol at $-20{ }^{\circ} \mathrm{C}$ for $10 \mathrm{~min}$, washed, and incubated in $2 \times \mathrm{SSC}$ $(0.3 \mathrm{M} \mathrm{NaCl}, 0.03 \mathrm{M}$ sodium citrate $(\mathrm{pH} 7 \cdot 0))$ for $20 \mathrm{~min}$. Fluorescent necrotic cells were visualized at excitation wavelength of $535 \mathrm{~nm}$ and using an emission filter of a wavelength $>605 \mathrm{~nm}$ and quantified the same way as Hoechst-stained samples.

\section{Reverse transcription}

Total RNA was isolated from astrocyte cultures using PeqGold RNA pure (PeqLab, Erlangen, Germany). The procedure was performed according to the instruction of the manufacturer. RNA concentration was measured photometrically using BioPhotometer (Eppendorf). RNA integrity was tested randomly by $1 \%(\mathrm{w} / \mathrm{v})$ agarose denaturing gel electrophoresis and ethidium bromide staining and visualized under UV-illumination. Firststrand complementary DNA (cDNA) was synthesized from $0.5 \mu \mathrm{g}$ total RNA. In brief, total RNA dissolved in $7 \mu \mathrm{l}$ diethyl pyrocarbonate water was pre-incubated at $85^{\circ} \mathrm{C}$ for $5 \mathrm{~min}$ and placed immediately on ice. Subsequently, the reaction buffer consisting of $8 \mathrm{U} / \mu \mathrm{l}$ M-MLV reverse transcriptase, $8 \mathrm{mM}$ dithiotreitol, $40 \mathrm{mM}$ Tris-HCl, $60 \mathrm{mM} \mathrm{KCl}$, and $2.4 \mathrm{mM} \mathrm{MgCl} 2$, and $0.4 \mathrm{mM}$ each dNTP (Roti-mix PCR3, Roth) was added to RNA giving a final volume of $12.5 \mu \mathrm{l}$. After $1 \mathrm{~h}$ incubation at $37^{\circ} \mathrm{C}$, reverse transcription was stopped by heatinactivating the enzyme at $70^{\circ} \mathrm{C}$ for $15 \mathrm{~min}$. Addition of water instead of RNA served as negative control. Transcripts of $18 \mathrm{~S}$ ribosomal RNA (18S rRNA) and hypoxanthine guanine phosphoribosyl transferase
(Hprt), spanning over intron-exon borders, served as control for RNA purity and normalization of sample quantity.

\section{Real time-PCR analysis}

Quantitative real-time PCR (RT-PCR) analysis of fusion and fission genes, pro-/anti-apoptotic, as well as proliferation markers was performed using SYBR Green technology and carried out by the iQ5 detection system (Bio-Rad). Forward and reverse primers (Table 1) for specific amplification of Mfn1, Mfn2, Fis 1, Dnm1l, a proliferation marker, proliferating cell nuclear antigen (Pcna), an anti-apoptotic marker, B-cell lymphoma protein 2 (Bcl2), and a pro-apoptotic marker, BCL2associated $\mathrm{X}$ protein $(B a x)$, were designed eliminating the possibility of amplifying genomic DNA. For each set of primers, a basic local alignment search tool (BLAST, NCBI) search revealed that sequence homology was obtained specifically for the target gene. The cDNA from untreated control and treated cell samples obtained after reverse transcription was diluted 1:10 and added to a solution containing $5 \mu \mathrm{M}$ primers and IQ SYBR Green Supermix (Bio-Rad) consisting of $25 \mathrm{U} / \mathrm{ml}$ iTaq polymerase, $50 \mathrm{mM} \mathrm{KCl}, 20 \mathrm{mM}$ Tris- $\mathrm{HCl}, 0.2 \mathrm{mM}$ each dNTP, $3 \mathrm{mM} \mathrm{MgCl} 2$, SYBR Green I, and stabilizers. The RT-PCR protocol was composed of an initial denaturation step for $3 \mathrm{~min}$ at $95^{\circ} \mathrm{C}$ followed by 40 cycles consisting of $10 \mathrm{~s}$ at $95^{\circ} \mathrm{C}, 30 \mathrm{~s}$ at the appropriate for the target gene annealing temperature $\left(53{ }^{\circ} \mathrm{C}-\right.$ Mfn1, Mfn2; $58^{\circ} \mathrm{C}-$ Fis 1, Dnm1l; $59^{\circ} \mathrm{C}-$ Bcl2, Bax, Pcna; $60^{\circ} \mathrm{C}-18 S$ rRNA; $\left.61^{\circ} \mathrm{C}-H p r t\right), 30 \mathrm{~s}$ at $72^{\circ} \mathrm{C}$, and $10 \mathrm{~s}$ at $78^{\circ} \mathrm{C}$. To obtain melting curves for the resulting PCR products, a final step was added to the RT-PCR consisting of 81 cycles of increasing temperature from $55^{\circ} \mathrm{C}$ to $81^{\circ} \mathrm{C}$ by $0.5{ }^{\circ} \mathrm{C}$ for $10 \mathrm{~s}$ each step. The PCR products were quantified using the relative $\Delta C_{\mathrm{t}}$ method. Relative quantification relates the PCR signal of the target transcript to that of $18 S \mathrm{rRNA}$ in treated cells with respect to untreated cells. A test for an approximately equal efficiency of target amplification was performed by looking at $\Delta C_{\mathrm{t}}$ value variations with template dilutions. 18S rRNA and Hprt served as endogenous control in the validation experiments. The absolute value of the slope of $\log$ input amounts versus $\Delta C_{\mathrm{t}}$ should be $\sim-3.3$ and the efficiency $\sim 100 \%$. The validation experiments passed this test. The results are expressed as an average of triplicate samples of at least three independent experiments for control and treated cells.

\section{Statistical analysis}

Data are presented as means \pm s.E.M. of at least three to five independent experiments. For statistical analysis, data were analyzed by ANOVA followed by a post hoc Tukey's test and regarded as statistically significant at $P \leq 0 \cdot 05$. 
Table 1 Information about primers used in the study

GenBank accession no.

NM_024200

Gene

Mfn $1^{\mathrm{a}}$

$\operatorname{Mfn} 2^{b}$

NM_133201

Dnm1F

NM_152816

Fis $1^{\mathrm{d}}$

NM_025562

$B c / 2^{e}$

BC089016

$\operatorname{Bax}^{\mathrm{f}}$

NM_007527

Pcna ${ }^{9}$

X53068

$18 S$ rRNA

X00686

Hprt $^{\text {h }}$

NC_000086.6
Primer sequences $5^{\prime}-3^{\prime}$

Product size (bp)

CAG AGA AGA GGG TTT ATT CA

ACT CAT CAA CCA AAA CAG AT

TGA ATG TTG TGT TCT TTC TG

AAG TGC TCT CTG CTA AAT GT

TTT GCT CGT GTG AAG ACT GG

TCC TGG AGC TTC CTT TCT GA

CTA CAG GGG TGC AGG AGA AA

AGA TGG ACT GGT AGG CAT GG

CAT CCC AGC TTC ACA TAA CC

GCA ATC CGA CTC ACC AAT AC

GGC AGA CAG TGA CCA TCT TT

AGG GGA CCT GAG GTT TAT TG

TCA GGA GCA ATC TTC AAA GG

TTT TTC ACA AAA GCC ACT CC

CGG CTA CCA CAT CCA AGG AA

GCT GGA ATT ACC GCG GCT

GCT GGT GAA AAG GAC CTC T

CAC AGG ACT AGA ACA CCT GC
180

175

175

228

194

186

248

${ }^{\mathrm{a}}$ Mitofusin 1.

${ }^{\mathrm{b}}$ Mitofusin 2.

${ }^{c}$ Dynamin-related protein 1.

dFission 1 (mitochondrial outer membrane) homolog.

${ }^{\mathrm{e}} \mathrm{B}$-cell lymphoma protein 2.

f Bcl2-associated X protein.

${ }^{\text {g}}$ Proliferating cell nuclear antigen.

${ }^{h}$ Hypoxanthine guanine phosphoribosyl transferase.

\section{Results}

\section{Gender-specific effects of steroid hormones on cell viability and apoptosis}

Cortical astroglial cell cultures prepared from postnatal mouse brains revealed a high yield $(>95 \%)$ of glial fibrillary acidic protein (GFAP)-positive cells, as determined by immunocytochemistry against the GFAP, an astrocyte-specific protein marker. Neurons, oligodendrocytes, and microglia were virtually absent from astrocyte cell cultures (Pawlak et al. 2005, Horvat et al. 2006). Astrocytes from both genders express $E R$ and $P R$ as studied by RT-PCR for ER $\alpha, \mathrm{ER} \beta$, and PR (data not shown).

In a first attempt, we have analyzed gender-specific effects of E- and P-treatment on proliferation and apoptotic death of cortical astrocytes. Female and male astrocyte cultures were treated with $10^{-7} \mathrm{M} \mathrm{E}$ or $\mathrm{P}$ in the absence or presence of the appropriate receptor inhibitor, i.e. ICI and Rti as inhibitors for ER and PR respectively. Viable, apoptotic, and necrotic astrocytes were distinguished by fluorescent labeling of cell nuclei with Hoechst 33342. Before and after treatments, no significant changes in the number of necrotic cells were detected $(0-2$ cells per total number of $\sim 100$ cells; data not shown). This finding was supported by experimental data obtained from propidium iodide staining that labels necrotic cells (data not shown). Due to the fact of a constant number of necrotic astrocytes, we focused on the determination of the number of viable and apoptotic astrocytes before and after hormone application. Treatment of cortical astrocytes from female brains with $\mathrm{E}$ or $\mathrm{P}$ for $24 \mathrm{~h}$ induced a significant increase in the number of Hoechst-stained viable cells by $\sim 50 \%$ (Fig. 1A). Co-application of $\mathrm{P}$ and $\mathrm{PR}$ inhibitor suppressed this effect, whereas the ER inhibitor did not affect the E-mediated effect (Fig. 1A). Independent of the steroid hormone applied, the number of apoptotic cells was not significantly changed in female astrocyte cultures (Fig. 1B). In contrast, the application of $\mathrm{E}$ did not exert any effect on the number of viable male cells, whereas $\mathrm{P}$ caused a decrease (Fig. 1C). The latter effect was counteracted by Rti. Compared with female astrocytes, opposite effects were observed concerning apoptosis of male astrocytes. Both hormones provoked a significant three- to fourfold induction that was partially suppressed by ICI and completely antagonized by Rti (Fig. 1D).

In the next step, we measured transcription levels of pro-, anti-apoptotic, and proliferation markers (Bax, Bcl2, and Pcna respectively) to figure out, whether changes in the viable and apoptotic cell numbers resemble changes in the marker gene expression. Primers used for RT-PCR amplification are given in Table 1 . They were designed as intron over-spanning sequences except for $18 S$ rRNA. Only single bands at correct molecular weight according to Table 1 were observed for the PCR products of the housekeeping gene Hprt and the genes of interest in an 
Female

A

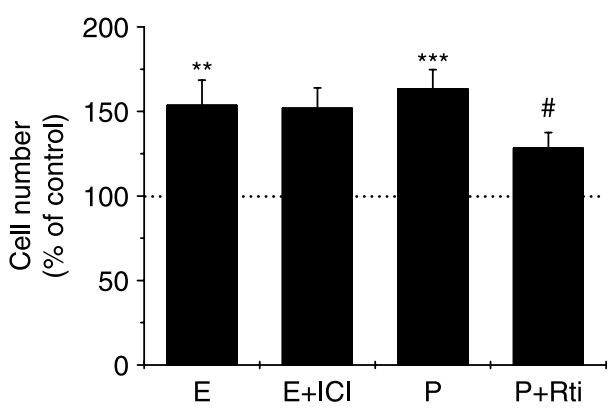

B

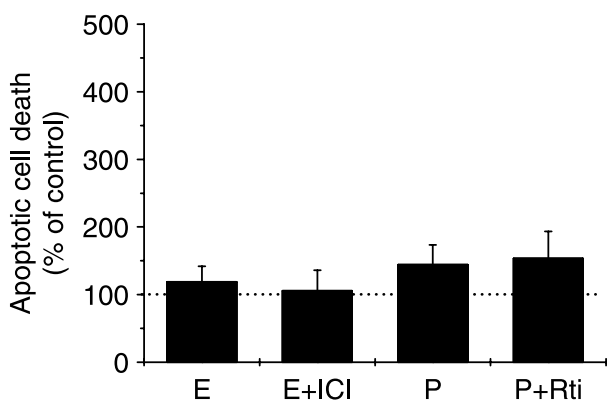

Male

C

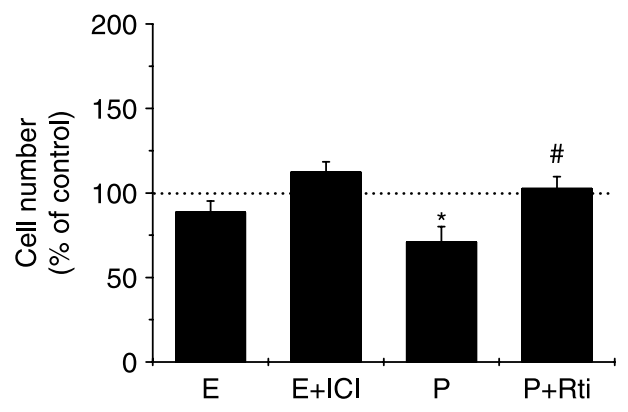

D

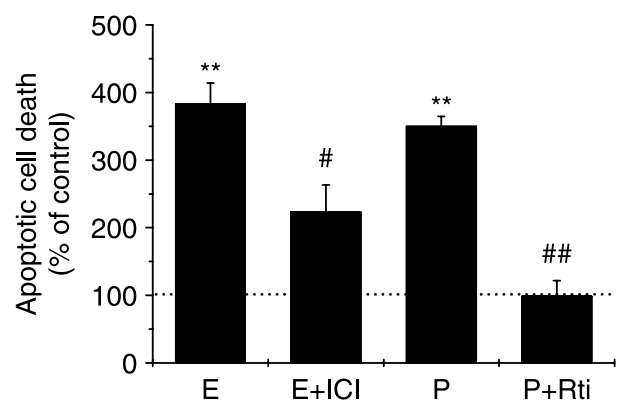

Figure 1 Effect of estrogen $(E)$ and progesterone $(P)$ on $(A$ and $C)$ the viability and $(B$ and $D)$ apoptotic cell death of female and male cortical astrocytes. Primary astrocyte cultures from (A and B) female and (C and D) male Balb/c mice were treated with $10^{-7} \mathrm{M} \mathrm{E}$ or $\mathrm{P}$ or co-treated with $10^{-6} \mathrm{M}$ of the appropriate inhibitors of steroid receptors ICI 182780 (ICl for ER) and Rti 3021-022 (Rti for PR) for $24 \mathrm{~h}$. The number of (A and C) viable astrocytes and (B and D) apoptotic cells were scored after staining astrocyte nuclei with Hoechst 33342. Data represent the percentage of the ratio of sample cell numbers to untreated control cell numbers referred to as $100 \%$ (dotted lines) as means \pm S.E.M. of triplicates of five independent experiments. ${ }^{\star} P<0.05,{ }^{* *} P<0.01$, ${ }^{\star * \star} P<0.001$, E-/P-treated samples versus untreated controls; ${ }^{\#} P<0.05$, ${ }^{\# \#} P<0.01$, inhibitor plus E-/P-treated samples versus E-/P-treated samples.

agarose gel. This indicates that the isolated RNA was free of genomic DNA impurities. In female cortical astrocytes, E exerted no significant effect on the transcription of anti- and pro-apoptotic marker genes, whereas $\mathrm{P}$ induced an approximately sixfold upregulation of $B c l 2$ and $B a x$ transcription (Fig. 2A), which was partially downregulated by Rti. Unlike females, male astrocytes responded to $\mathrm{E}$ application with an increased transcription of the apoptotic marker Bax and a decreased transcription of anti-apoptotic Bcl2 (Fig. 2C). Treatment of male astrocytes with $\mathrm{P}$ caused a decreased transcription of both marker genes. The $\mathrm{P}$ effect was completely inhibited by Rti, whereas ICI reversed only the E effect on $B c l 2$ transcription.

In female astrocytes, Pcna transcription was five- to eightfold upregulated, irrespective of the hormone (Fig. 2B). Again, ICI did not prevent the E effect, but Rti partially abolished the $\mathrm{P}$ effect. Pcna transcription levels in male astrocytes were stimulated by $\mathrm{E}$, but significantly downregulated by P (Fig. 2D). ICI showed no effect. Here again, Rti antagonized the effect.

\section{Gender-specific effects of steroid hormones on fusion and fission gene transcription}

After treatment of female and male cortical astrocytes with E or P, the transcription levels of $M f n 1$, Mfn2, Fis1, and Dnm1l were determined by RT-PCR (Table 1, Fig. 3). In female astrocytes, E stimulated $M f n 2$ but not $M f n 1$ transcription, whereas $\mathrm{P}$ exposure caused an increase in both fusion transcripts (Fig. 3A). In male astrocytes, E decreased $M f n 1$ but increased $M f n 2$ transcription, whereas $\mathrm{P}$ downregulated both fusion gene transcript levels (Fig. 3C). Regarding fission gene transcription, E and $\mathrm{P}$ stimulated both, Dnm 1l and Fis1, in female astrocytes (Fig. 3B). In male astrocytes, E also increased Dnm 1 land Fis 1 transcription, but P decreased the transcription of both fission genes (Fig. 3D). Here again, ICI did not antagonize the $\mathrm{E}$ effects in female astrocytes, whereas Rti blocked partially the $\mathrm{P}$ effect (Fig. 3A and B). In male astrocytes, ICI blocked only the E effect on $M f n 1$ transcription, and Rti reversed the $\mathrm{P}$ effect on the transcription of all fusion and fission genes (Fig. 3C and D). 
Female

A

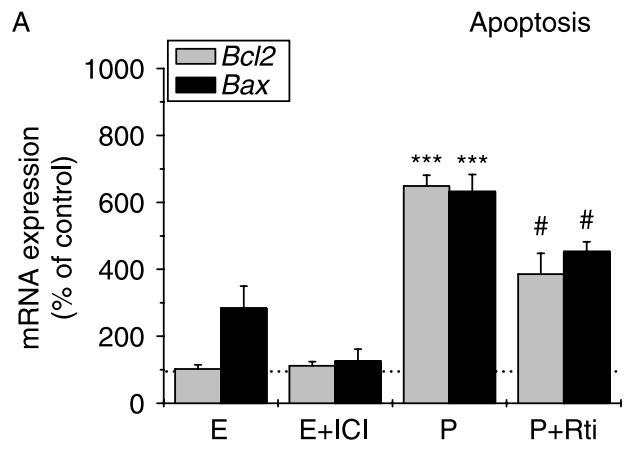

B

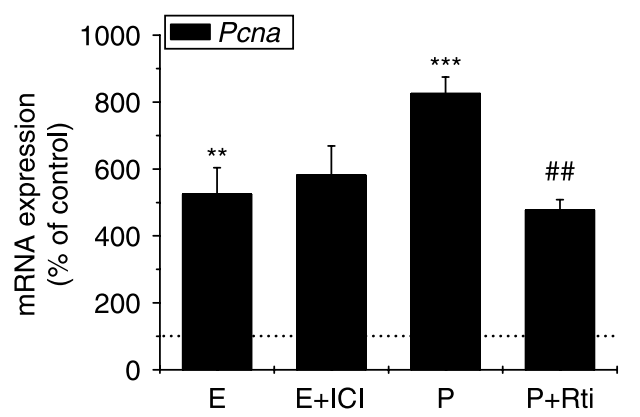

Male

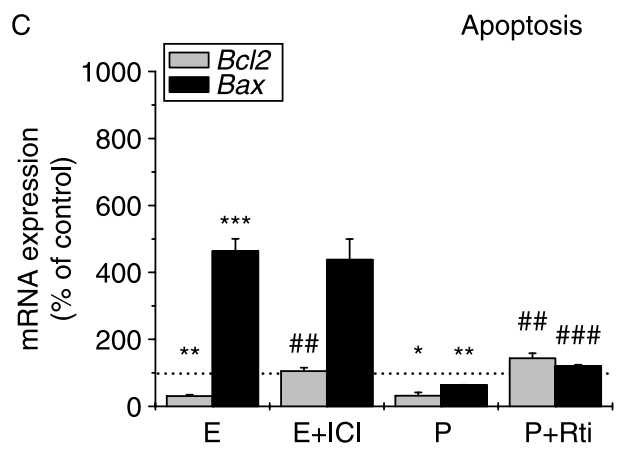

D

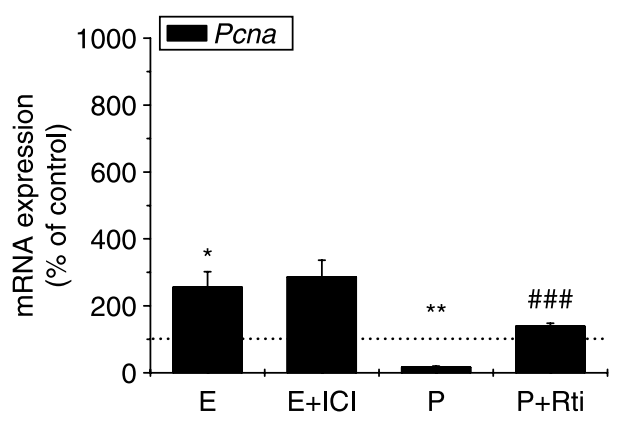

Figure 2 Effect of estrogen $(E)$ and progesterone $(P)$ on the transcription of apoptosis and proliferation markers in cortical astrocytes. Cultured astrocytes from ( $A$ and $B$ ) female and $(C$ and $D)$ male brains were treated with $10^{-7} \mathrm{M} \mathrm{E}$ or $\mathrm{P}$ in the absence or presence of $10^{-6} \mathrm{M} \mathrm{ICl}$ or Rti for $24 \mathrm{~h}$. Specific quantification of $B c / 2$ ( $A$ and $C$; grey columns) and Bax transcripts ( $A$ and $C$; black columns) as well as Pcna transcripts (B and D) was performed by RT-PCR. Data were normalized to $18 S$ rRNA and Hprt. Untreated astrocytes from the same cell culture preparation served as negative control and were set $100 \%$ (dotted lines). Data represent the percentage of control expressed as means \pm S.E.M. of triplicates of five independent experiments. ${ }^{\star} P<0.05,{ }^{\star \star} P<0.01,{ }^{\star \star \star} P<0.001$, E-/P-treated samples versus untreated controls; ${ }^{\#} P<0.05,{ }^{\# \#} P<0.01,{ }^{\# \# \#} P<0.001$, inhibitor plus E-/P-treated samples versus E-/P-treated samples.

Correlation analyses of $M f n 1$ with $B c l 2$ transcripts and Dnm $1 l$ with Pcna for female and male astrocytes are given in Fig. 4A-D. It becomes evident that $M f n 1$ correlates well with Bcl2 and Dnm1l with Pcna for both steroid hormones and both genders (Fig. $4 \mathrm{~A}$ and $\mathrm{C}$ as well as $\mathrm{B}$ and D respectively) by showing similar transcription levels. Several observations concerning gender-specific differences of the hormonal regulation deserve attention: i) In females, P increased $M f n 1, B c l 2$, and Dnm1l, Pcna transcript levels (Fig. 4A and B); in males, gene transcription was decreased (Fig. 4Cand D). ii) Treatment of astrocytes with E caused higher transcript levels of $M f n 1$, Bcl2and Dnm1l, Pcnain females than in males (Fig. 4A and $\mathrm{B}$ versus $\mathrm{C}$ and $\mathrm{D})$. In females, $M f n 1$ and $B c l 2$ are not changed by E, but decreased in males. Dnm1land Pcna are increased by $\mathrm{E}$ in females to a greater extent than in males.

Our data suggest that Dnm $1 l$ correlates with proliferation and Fis 1 with apoptosis in both genders. Therefore, we attempted to correlate the ratios of $\mathrm{Dnm} 1 \mathrm{l} / \mathrm{Fis} 1$ and Pcna/Bax transcription with viable/apoptotic cell number as shown in Fig. 5A (female) and B (male). The following gender-specific differences become obvious when comparing all three ratios: i) The Pcna/Bax transcript level and viable/apoptotic cell ratios are $>1$ for female astrocytes treated with $\mathrm{E}$ or $\mathrm{P}$, whereas these levels are $<1$ for $\mathrm{E}$-/P-treated male astrocytes. ii) The Dnm 1l/Fis1 ratios are decreased in P-versus E-treated male astrocytes, but slightly increased in females. For both genders, i) the ratios of Pcna/Bax are decreased in P-treated compared with E-treated cells; ii) the ratios of viable/apoptotic cell numbers do not differ between the two steroid treatments.

\section{Discussion}

Astrocytes are essential for the support of neurons in the CNS (Pellerin et al. 2007, Schousboe et al. 2007). They are implicated in the regulation of growth, cell proliferation, and neuroprotection in the brain (Beyer 1999, Beyer et al. 2003, Garcia-Segura et al. 2003, Kajta \& Beyer 2003, Kipp et al. 2006, McCarthy 2008). In the past years, the neuroprotective aspects of $\mathrm{E}$ and $\mathrm{P}$ effects in the CNS have been analyzed using in vitro and in vivo 


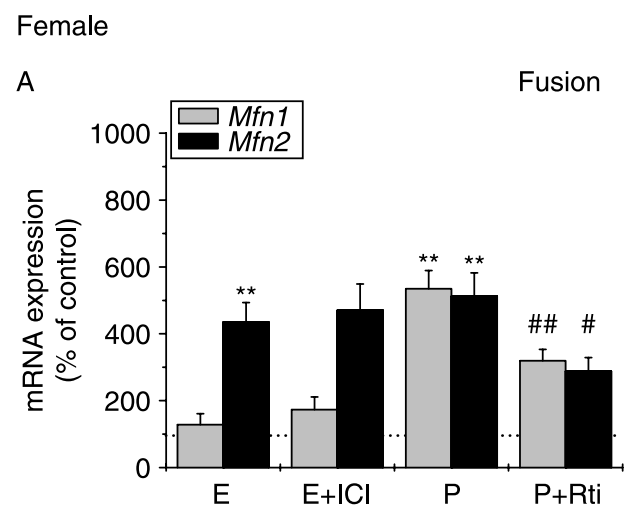

Male

B

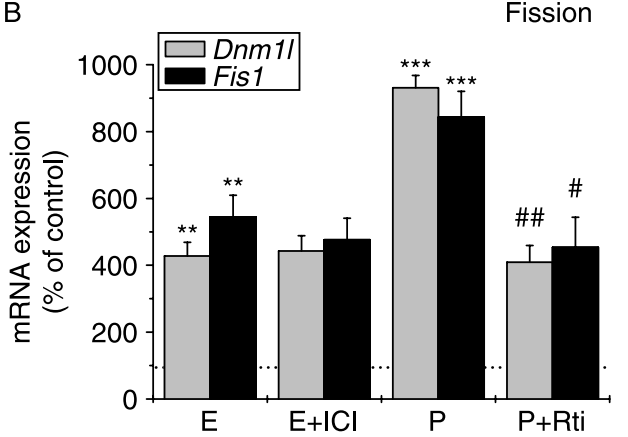

C

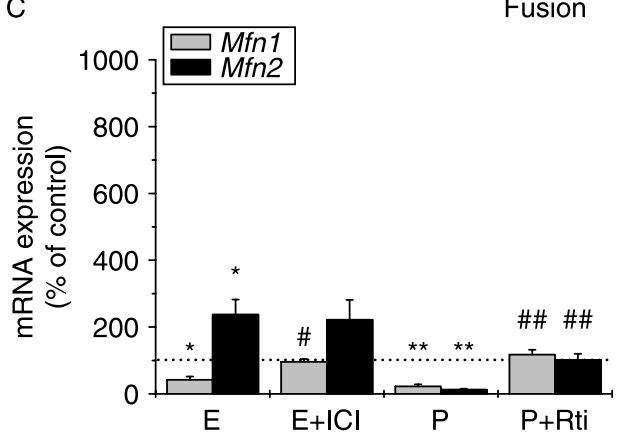

Fission

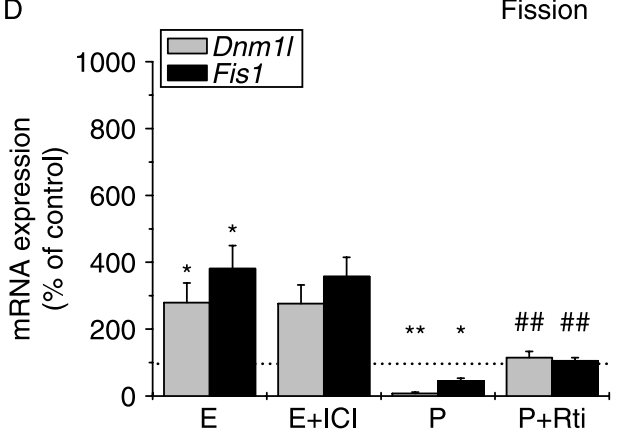

Figure 3 Effect of estrogen $(E)$ and progesterone $(P)$ on the transcription of mitochondrial fusion and fission genes in cortical astrocytes from female and male brains. Primary cortical astrocytes from (A and $B)$ female and $(C$ and $D)$ male mouse brains were incubated in the presence of $10^{-7} \mathrm{M} \mathrm{E}$ or $\mathrm{P}$ for $24 \mathrm{~h}$. Antagonists of estrogen and progesterone receptors ( $\mathrm{ICl}$ and Rti respectively) were added simultaneously to the respective steroid hormone treatment. Specific quantification of fusion genes, Mfn1 (A and C; grey columns) and Mfn2 (A and C; black columns), and fission genes, Dnm1I (B and D; grey columns) and Fis1 (B and D; black columns), was performed by RT-PCR. Data were normalized to $18 S$ rRNA and Hprt and related to the transcription levels in untreated control astrocytes set $100 \%$ (dotted lines). Data represent the percentage of control as means \pm S.E.M. of triplicates of five independent experiments. ${ }^{\star} P<0.05$, ${ }^{\star \star} P<0.01$, ${ }^{\star \star \star} P<0.001$, E-/P-treated samples versus untreated controls; ${ }^{\#} P<0.05$, ${ }^{\# \#} P<0.01$, inhibitor plus E-/Ptreated samples versus E-/P-treated samples.

approaches. Besides direct interactions of $\mathrm{E}$ and $\mathrm{P}$ with neurons (Kipp et al. 2006, Stein et al. 2008), there are indications that neurons are not always protected directly. However, E has consistently been demonstrated to be protective in vivo and in organotypical cultures (Dhandapani \& Brann 2002, Simpkins et al. 2005). This suggests the involvement of another nonneuronal brain cell type exerting a protective function. Of the non-neuronal brain cell types, astrocytes have the greatest potential for a possible involvement in the mediation of steroid-mediated neuroprotective effects. Astrocytes are the most abundant type of glial cells in the brain and are located juxtaposition to neurons, outnumbering them by a 10:1 ratio in the cortex. This cell type has the greatest potential for a critical role in steroid-mediated protection of neurons in the brain, since ablation of astrocytes in vivo results in a significant decrease in neuronal survival (Cui et al. 2001). Indeed, astrocytes are well-described target cells for $\mathrm{E}$ and $\mathrm{P}$ expressing the corresponding nuclear steroid receptors
(Jung Testas et al. 1992, Santagati et al. 1994, Beyer 1999, Quadros et al. 2007). Confirming previously published data (Pawlak et al. 2005), we observed transcripts for $\mathrm{ER} \alpha, \mathrm{ER} \beta$, and PR in cortical astrocytes from both genders.

Besides the above described supportive function, astrocytes play a major role for the energy homeostasis in the brain pointing at astrocyte mitochondria as an interesting compartment involved in neuroprotection. However, there is only limited information available on interactions between sex steroids and mitochondria. In recent studies, we and others have shown that $\mathrm{E}$ promotes the expression of mitochondria-encoded subunits of the respiratory chain, thereby influencing mitochondrial respiratory activity and enhancing functional efficiency (Araújo et al. 2008, Irwin et al. 2008). Mitochondrial functions are not only affected by regulation of nuclear and mitochondrial gene expression and activity of the respiratory chain (Zheng \& Ramirez 1999, Araújo et al. 2008, Irwin et al. 2008), but 

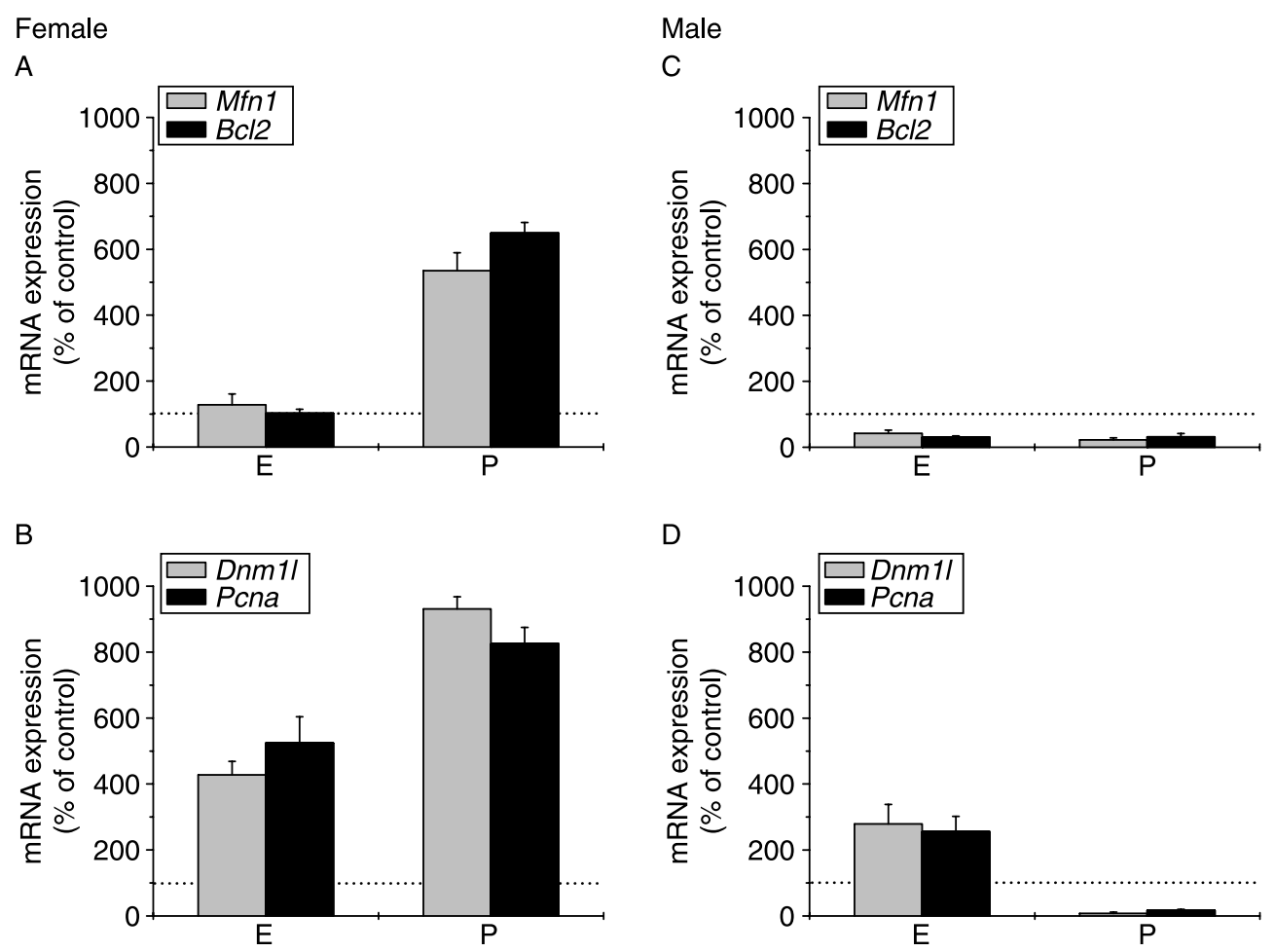

Figure 4 Correlation analysis between (A and C) Mfn1 and Bcl2 gene transcription as well as (B and D) Dnm1I and $P$ cna gene transcription in $(A$ and $B)$ female and $(C$ and $D)$ male astrocytes. Data used for this graph derived from Figs 1-3. Gene transcription levels for Mfn1 (A and C; grey columns) and Bcl2 (A and C; black columns) as well as for Dnm1/ (B and D; grey columns), and Pcna (B and D; black columns) after E-/P-treatment in the absence or presence of $\mathrm{ICl} / \mathrm{R}$ ti for $24 \mathrm{~h}$ are presented as percentage of control set $100 \%$ (dotted lines). Note that $P$ exerted opposite effects in female and male astrocytes. Treatment of astrocytes with $E$ caused higher transcription levels of all four genes in $(A$ and $B)$ females compared with $(C$ and $D)$ males.

also by the morphology of these organelles. It is noteworthy that $\mathrm{E}$ influences mitochondria not only through its nuclear receptors, but acts also directly by binding of $\mathrm{E}$ to mitochondria in vitro and in situ (Yang et al. 2004, Stirone et al. 2005, Yager \& Chen 2007, Milner et al. 2008). Interestingly, we observed that some effects, in particular those exerted by $\mathrm{E}$ but not by $\mathrm{P}$, were not subject to inhibition by classical nuclear ER antagonists. Proliferation and fission gene transcription were obviously mediated by E via non-classical mechanisms in male and female astrocytes. The context of mitochondrial fission and cell division additionally supports the role of one for the other. Such a proliferative E action that is intracellularly transmitted through, for instance, the MAP kinase system has already been reported for non-neural cells (Bouskine et al. 2008). This is in line with a previous observation of a rapid activation of ERK in astroglia (Ivanova et al. 2001). Intriguingly, a recent report suggests a mitochondrial ER-mediated pathway in stimulation of cell division (Chen et al. 2008). Besides proliferation, the ER antagonist ICI did not abolish any of the E effects on female astrocytes studied, whereas in male astrocytes, ICI prevented the $\mathrm{E}$ effects by decreasing apoptotic cell death and increasing $B c l 2$ and $M f n 1$ transcription. This indicates gender-specific $\mathrm{E}$ actions in the context of anti-apoptotic processes and an involvement of $M f n 1$ therein.

It is known for several years and for a wide range of neurodegenerative disorders that they show distinct sex-specific differences in course and incidence. Thus, a female prevalence is known for Alzheimer's disease and multiple sclerosis, whereas a male prevalence was observed for Parkinson's disease and stroke. In particular, intact adult female rodents sustain lower mortality and less neuronal damage when compared with age-matched male rodents following middle cerebral artery occlusion (Alkayed et al. 1998). Parkinson's disease reveals a clear gender disparity (Shulman 2007). Interestingly, E appears to exert opposite effects in females and males in an experimental animal model for Parkinson's disease being neuroprotective only in females (Gillies et al. 2004). Estrogen replacement therapy leads to an improvement of Parkinsonian symptoms of women in the menopause (Shulman 2007). Both disorders, stroke and Parkinson's disease, and also Alzheimer's disease are 
Female

A

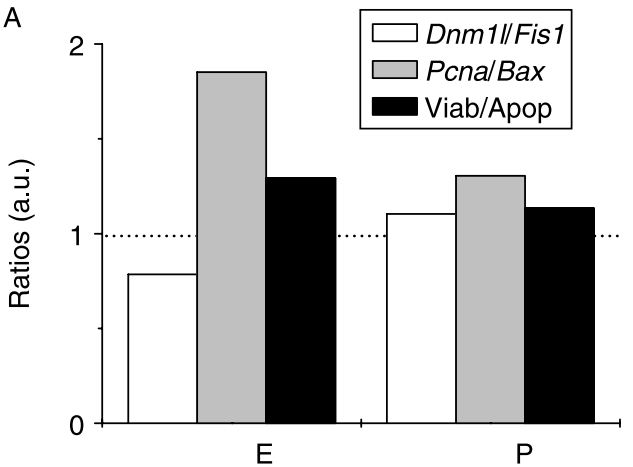

Male

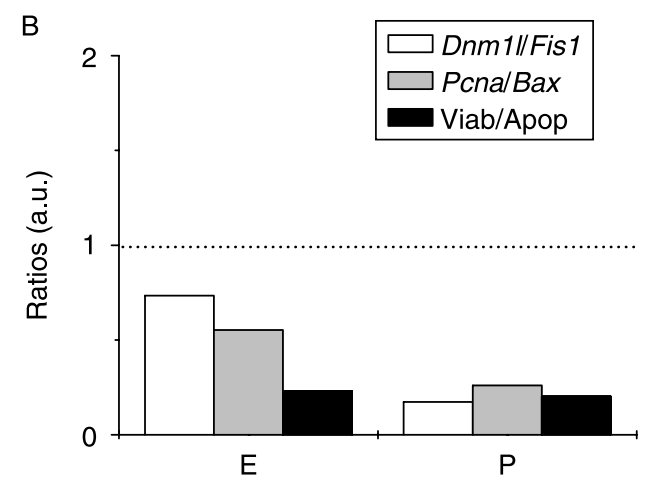

Figure 5 Correlation analysis between the ratios of Dnm1//Fis 1 transcription levels (Dnm1//Fis1), Pcna/Bax transcription levels (Pcna/Bax), and viable/apoptotic cell numbers (Viab/Apop) for (A) female and (B) male astrocytes. Gender-specific differences become obvious when comparing the ratios of Dnm1/to Fis 1 (white columns) with those of $P$ cna to Bax (grey columns) or with the ratios of viable to apoptotic cell number (black columns). Note that the Pcna/Bax transcripts and viable/apoptotic cell ratios are $>1$ (dotted line) for $(A)$ female astrocytes treated with $E$ or $P$, whereas these levels are $<1$ (dotted line) for $(B) E$-/P-treated male astrocytes. For both, (A) female and (B) male astrocytes, the ratios of Pcna/Bax are decreased in $\mathrm{P}$-treated compared with E-treated cells, whereas the Dnm1//Fis1 ratios are also decreased in P-versus E-treated male astrocytes, but marginally increased in females. The ratios of viable/apoptotic cell numbers do not differ between the two steroid treatments neither in females (A) nor in males (B). a.u., arbitrary units.

characterized by a disruption of mitochondrial function (Singh et al. 2006, Thomas \& Beal 2007, Poole et al. 2008, Schapira 2008). This raised the question whether steroid-mediated regulation of mitochondrial morphology and function could be involved in the gender specificity of neurodegenerative and neuroprotective processes. Besides functional characteristics, such as energy production, the morphology of mitochondria including the expression of fusion and fission genes are indicators of mitochondrial vitality. Elevated fusion processes cause an elongation of mitochondria, whereas an increase in fission leads to mitochondrial fragmentation (Chan 2007). Generally, an imbalance of mitochondrial fusion and fission causes mitochondrial and cellular dysfunction indicating that fusion and fission processes are essentially linked to cell function and protection/apoptosis. In an attempt to better understand the protective action of $\mathrm{E}$ and $\mathrm{P}$, we have focused on fusion and fission processes in astrocytes and have tried to correlate their regulation with cell viability and apoptosis.

Cultured cortical astrocytes were treated with $\mathrm{E}$ concentrations known to be effective in the brain (Luconi et al. 1999, Ivanova et al. 2001, Pawlak et al. 2005, Rune et al. 2006, von Schassen et al. 2006, Araújo et al. 2008). Under basal (physiological) conditions, cultured cortical astrocytes from males and females did not differ in their characteristics for mitochondria morphology and cell viability. E appeared to stimulate proliferation in females, whereas $P$ facilitates cell death in males. The age-related drop of $E$ levels in females may, thus, play a role for the vulnerability of cortical neurons in late-onset neurodegenerative disorders, such as Alzheimer's disease, due to reduced astrocyte stimulation and survival. We might speculate that the prevalence for Alzheimer's disease observed for females is causally linked to decreased $\mathrm{E}$ levels and astrocyte activation. Notwithstanding the importance of our observations, we did not provide a pathological model with our experimental set-up.

For E-treated astrocytes of both genders, we observed a correlation between $M f n 1$ and anti-apoptotic marker Bcl2 transcription as well as Dnm1l and proliferation marker Pcna transcription. This indicates a role of $M f n 1$ in anti-apoptotic processes and Dnm1l in cell proliferation and is in agreement with the generally accepted role of fusion genes in anti-apoptotic processes and fission genes in proliferation and apoptosis (Yaffe 1999, James et al. 2003, Karbowski \& Youle 2003, Olichon et al. 2003, Yoon et al. 2003, Lee et al. 2004, Stojanovski et al. 2004, Chen \& Chan 2005, Chen et al. 2005, Chan 2007). Our studies also revealed gender differences in the transcription of $M f n 1$ and Dnmll showing higher levels in females that were accompanied by lower apoptotic cell death and elevated number of viable cells. The role of Dnm 1 lin mitochondrial fragmentation was previously supported by the discoveries that Dnm1l mutations blocked and Dnm1l overexpression increased mitochondrial proliferation (Bleazard et al. 1999, Labrousse et al. 1999). Furthermore, Dnm1l was co-localized with constrictions in mitochondria that can coincide with actual division events (Bleazard et al. 1999, Labrousse et al. 1999). During mitosis, Dnm1lis involved in fission of mitochondria, a phenomenon that might facilitate the 
partitioning of fragmented mitochondria to daughter cells during cytokinesis (Taguchi et al. 2007). This makes Dnm 1 la factor of controlling mitochondrial division and a potential candidate for steroid-mediated regulation of cell proliferation, although the involvement of $\mathrm{Dnm} 1 \mathrm{l}$ in apoptosis cannot completely been ruled out (Frank et al. 2001). We have deliberately chosen the Dnm1l/Fis 1 ratio to describe proliferative and apoptotic events in astrocyte cultures. This enables us to discriminate both opposite mechanisms in cultures of equal numbers of viable cells. A constant number of cells does not allow to conclude whether there is an effect or no effect on both processes by hormones.

Comparing the anti- and pro-apoptotic marker gene transcription with apoptotic cell death, it becomes obvious that an increase or the absence of differences in Bax compared with $B c l 2$ is related to increased or unchanged apoptosis respectively. The apparent discrepancy between the effect of $\mathrm{P}$ on male astrocytes showing a reduced Bax transcription and increased apoptotic cell death can be clarified by determination of the $B a x / B c l 2$ ratio which is elevated. This points at an increase of apoptotic cell death, although the individual values of $B a x$ and $B c l 2$ in males are below the basal levels found in controls.

A clear gender-specific difference in the apoptosis rate of steroid-treated astrocytes was observed and could be correlated with fusion/fission gene transcription. Female astrocytes showed a mainly balanced increase of both, fusion and fission gene transcription. This can be considered as a reason for a balanced transcription of anti- and pro-apoptotic marker genes. On the contrary, fusion and fission gene transcription in males was imbalanced. Consequently, this imbalance caused a disturbance of the balance between anti- and pro-apoptotic marker genes, thus promoting apoptotic processes specifically in male astrocytes treated with gonadal steroids. A higher level of fusion gene transcription supports the increased astrocyte viability in females (Zuchner et al. 2004, Kijima et al. 2005). This could be, at least in part, due to the role of mitochondrial fusion processes in mixing mitochondrial DNA copies, thereby keeping high loads of mutated mitochondrial DNA below the threshold of developing diseases (Chen \& Chan 2005, Chan 2007).

The increased apoptosis in male astrocyte cultures could only be compensated by an elevation of proliferation resulting in an unchanged level of viable cells after steroid hormone treatment. Indeed, E-treated male astrocytes exhibited a higher Pcna/Bax ratio than P-treated cells. A ratio of Pcna/Bax $>1$ together with an increased proliferation marker transcription at constant levels of apoptosis in female astrocytes after steroid treatment caused an elevated number of viable cells. In contrast, male astrocytes demonstrated an increased proliferation after $\mathrm{E}$ treatment that compensated for the increased apoptosis, thereby demonstrating a similar number of viable cells as in untreated controls. However, a decreased proliferation after $\mathrm{P}$ treatment of male astrocytes was not able to counterbalance the increased apoptotic cell death leading to a net decrease in viable cells. Other authors have similarly demonstrated $\mathrm{E}$ to increase glial cell proliferation (Jung Testas et al. 1992, Dhandapani \& Brann 2002), whereas no effect of $P$ on cell proliferation was described. This could be due to the fact that genderspecific differences observed for P-treated astrocytes are counterbalanced in cell cultures of mixed sexes.

In conclusion, our data show for the first time that sex steroids are capable of influencing fusion and fission processes in astroglia mitochondria. Importantly, this effect occurred in a gender-specific way and revealed distinct differences in the effectiveness between $\mathrm{E}$ and P. Under physiological conditions, sex steroids may be important for the balance of mitochondrial morphology and function in the brain. After toxic events and under neuropathological conditions, sex steroids may differently contribute to the homeostasis and integrity of cell function, thus providing a basis for the well-known gender differences in neuronal vulnerability and cell death. Our findings suggest a pronounced sensitivity of male astroglia for apoptosis. This may serve as an explanation for the higher incidences of neurodegenerative diseases in males (Miller et al. 1998, Roof \& Hall 2000, van den Eeden et al. 2003, Dluzen \& McDermott 2004, Kenchappa et al. 2004, Baba et al. 2005, Marchetti et al. 2005).

\section{Declaration of interest}

The authors declare that there is no conflict of interest that could be perceived as prejudicing the impartiality of the research reported.

\section{Funding}

This work was supported by the Emmy-Noether-Programm of the Deutsche Forschungsgemeinschaft (S A), the START-Programm (S A) and IZKF BIOMAT (S A, C B) of the Faculty of Medicine, RWTH Aachen University.

\section{Acknowledgements}

We thank Petra Ibold for excellent technical support.

\section{References}

Alexander C, Votruba M, Pesch UE, Thiselton DL, Mayer S, Moore A, Rodriguez M, Kellner U, Leo-Kottler B, Auburger G et al. 2000 OPA1, encoding a dynamin-related GTPase, is mutated in autosomal dominant optic atrophy linked to chromosome $3 q 28$. Nature Genetics 26 211-215. 
Alkayed NJ, Harukuni I, Kimes AS, London ED, Traystman RJ \& Hurn PD 1998 Gender-linked brain injury in experimental stroke. Stroke 29 159-166.

Araújo GW, Beyer C \& Arnold S 2008 Oestrogen influences on mitochondrial gene expression and respiratory chain activity in cortical and mesencephalic astrocytes. Journal of Neuroendocrinology 20 930-941.

Arnold S 2005 Estrogen suppresses the impact of glucose deprivation on astrocytic calcium levels and signaling independently of the nuclear estrogen receptor. Neurobiology of Disease 20 82-92.

Azcoitia I, Garcia-Ovejero D, Chowen JA \& Garcia-Segura LM 2001 Astroglia play a key role in the neuroprotective actions of estrogen. Progress in Brain Research 132 469-478.

Baba Y, Putzke JD, Whaley NR, Wszolek ZK \& Uitti RJ 2005 Gender and the Parkinson's disease phenotype. Journal of Neurology 252 1201-1205.

Bakeeva LE, Chentsov YS \& Skulachev VP 1981 Ontogenesis of mitochondrial reticulum in rat diaphragm muscle. European Journal of Cell Biology 25 175-181.

Beyer C 1999 Estrogen and the developing mammalian brain. Anatomy and Embryology 199 379-390.

Beyer C, Pilgrim C \& Reisert I 1991 Dopamine content and metabolism in mesencephalic and diencephalic cell cultures: sex differences and effects of sex steroids. Journal of Neuroscience 11 1325-1333.

Beyer C, Pawlak J, Brito V, Karolczak M, Ivanova T \& Kuppers E 2003 Regulation of gene expression in the developing midbrain by estrogen: implication of classical and nonclassical steroid signaling. Annals of the New York Academy of Sciences 1007 17-28.

Bleazard W, McCaffery JM, King EJ, Bale S, Mozdy A, Tieu Q, Nunnari J \& Shaw JM 1999 The dynamin-related GTPase Dnm1 regulates mitochondrial fission in yeast. Nature Cell Biology 1 298-304.

Bouskine A, Nebout M, Mograbi B, Brücker-Davis F, Roger C \& Fenichel P 2008 Estrogens promote human testicular germ cell cancer through a membrane-mediated activation of extracellular regulated kinase and protein kinase A. Endocrinology 149 565-573.

Chan DC 2007 Mitochondrial dynamics in disease. New England Journal of Medicine 356 1707-1709.

Chen H \& Chan DC 2005 Emerging functions of mammalian mitochondrial fusion and fission. Human Molecular Genetics 14 R283-R289.

Chen H, Detmer SA, Ewald AJ, Griffin EE, Fraser SE \& Chan DC 2003 Mitofusins Mfn1 and Mfn2 coordinately regulate mitochondrial fusion and are essential for embryonic development. Journal of Cell Biology 160 189-200.

Chen H, Chomyn A \& Chan DC 2005 Disruption of fusion results in mitochondrial heterogeneity and dysfunction. Journal of Biological Chemistry 280 26185-26192.

Chen JQ, Brown TR \& Yager JD 2008 Mechanisms of hormone carcionogenesis: evolution of views, role of mitochondria. Advances in Experimental Medicine and Biology 630 1-18.

Cipolat S, Martins de Brito O, Dal Zilio B \& Scorrano L 2004 OPA1 requires mitofusin 1 to promote mitochondrial fusion. PNAS 101 15927-15932.

Cui W, Allen ND, Skynner M, Gusterson B \& Clark AJ 2001 Inducible ablation of astrocytes shows that these cells are required for neuronal survival in the adult brain. Glia 34 272-282.

Delettre C, Lenaers G, Griffoin JM, Gigarel N, Lorenzo C, Belenguer P, Pelloquin L, Grosgeorge J, Turc-Carel C, Perret E et al. 2000 Nuclear gene OPAl, encoding a mitochondrial dynamin-related protein, is mutated in dominant optic atrophy. Nature Genetics 26 207-210.

Detmer SA \& Chan DC 2007 a Complementation between mouse Mfn1 and Mfn2 protects mitochondrial fusion defects caused by CMT2A disease mutations. Journal of Cell Biology 176 405-414.

Detmer SA \& Chan DC $2007 b$ Functions and dysfunctions of mitochondrial dynamics. Nature Reviews. Molecular Cell Biology 8 870-879.

Dhandapani KM \& Brann DW 2002 Estrogen-astrocyte interactions: implications for neuroprotection. BMC Neuroscience 3 6-9.
Dluzen DE \& McDermott JL 2004 Developmental and genetic influences upon gender differences in methamphetamine-induced nigrostriatal dopaminergic neurotoxicity. Annals of the New York Academy of Sciences 1025 205-220.

van den Eeden SK, Tanner CM, Bernstein AL, Fross RD, Leimpeter A, Bloch DA \& Nelson LM 2003 Incidence of Parkinson's disease: variation by age, gender, and race/ethnicity. American Journal of Epidemiology 157 1015-1022.

Frank S, Gaume B, Bergmann-Leitner ES, Leitner WW, Robert EG, Catez F, Smith CL \& Youle RJ 2001 The role of dynamin-related protein 1, a mediator of mitochondrial fission, in apoptosis. Developmental Cell 1 515-525.

Garcia-Segura LM \& Melcangi RC 2006 Steroids and glial cell function. Glia 54 485-498.

Garcia-Segura LM, Veiga S, Sierra A, Melcangi RC \& Azcoitia I 2003 Aromatase: a neuroprotective enzyme. Progress in Neurobiology 71 31-41.

Gillies GE, Murray HE, Dexter D \& McArthur S 2004 Sex dimorphisms in the neuroprotective effects of estrogen in an animal model of Parkinson's disease. Pharmacology, Biochemistry, and Behavior 78 513-522.

Griparic L, van der Wel NN, Orozco IJ, Peters PJ \& van der Bliek AM 2004 Loss of the intermembrane space protein Mgm1/OPA1 induces swelling and localized constrictions along the lengths of mitochondria. Journal of Biological Chemistry 279 18792-18798.

Horvat S, Beyer C \& Arnold S 2006 Effect of hypoxia on the transcription pattern of subunit isoforms and the kinetics of cytochrome $c$ oxidase in cortical astrocytes and cerebellar neurons. Journal of Neurochemistry 99 937-951.

Irwin RW, Yao J, Hamilton RT, Cadenas E, Brinton RD \& Nilsen J 2008 Progesterone and estrogen regulate oxidative metabolism in brain mitochondria. Endocrinology 149 3167-3175.

Ivanova T, Koralczak M \& Beyer C 2001 Estrogen stimulates the mitogen-activated protein kinase pathway in midbrain astroglia. Brain Research 889 264-269.

James DI, Parone PA, Mattenberger Y \& Martinou JC 2003 hFis1, a novel component of the mammalian mitochondrial fission machinery. Journal of Biological Chemistry 278 36373-36379.

Jung Testas I, Renoir M, Bugnard H, Greene GL \& Baulien EE 1992 Demonstration of steroid hormone receptors and steroid action in primary cultures of rat glial cells. Journal of Steroid Biochemistry and Molecular Biology 41 621-631.

Kajta M \& Beyer C 2003 Cellular strategies of estrogen-mediated neuroprotection during brain development. Endocrine 21 3-9.

Karbowski M \& Youle RJ 2003 Dynamics of mitochondrial morphology in healthy cells and during apoptosis. Cell Death and Differentiation 10 870-880.

Kenchappa RS, Diwakar L, Annepu J \& Ravindranath V 2004 Estrogen and neuroprotection: higher constitutive expression of glutaredoxin in female mice offers protection against MPTP-mediated neurodegeneration. FASEB Journal 18 1102-1104.

Kijima K, Numakura C, Izumino H, Umetsu K, Nezu A, Shiiki T, Ogawa M, Ishizaki Y, Kitamura T, Shozawa Y et al. 2005 Mitochondrial GTPase mitofusin 2 mutation in Charcot-Marie-Tooth neuropathy type 2A. Human Genetics 116 23-27.

Kipp M, Karakaya S, Pawlak J, Araujo-Wright G, Arnold S \& Beyer C 2006 Estrogen and the development and protection of nigrostriatal dopaminergic neurons: concerted action of a multitude of signals, protective molecules, and growth factors. Frontiers in Neuroendocrinology 27 376-390.

Labrousse AM, Zapaterra M, Rube DA \& van der Bliek AM 1999 C. elegans dynamin-related protein $d r p-1$ controls severing of the mitochondrial outer membrane. Molecular Cell 4 815-826.

Lee YJ, Jeong SY, Karbowski M, Smith CL \& Youle RJ 2004 Roles of the mammalian mitochondrial fission and fusion mediators Fis1, Drp1, and Opal in apoptosis. Molecular Biology of the Cell 15 5001-5011.

Leonelli E, Bianchi R, Cavaletti G, Caruso D, Crippa D, Garcia-Segura LM, Lauria G, Magnaghi V, Roglio I \& Melcangi RC 2007 
Progesterone and its derivatives are neuroprotective agents in experimental diabetic neuropathy: a multimodal analysis. Neuroscience 144 1293-1304.

Luconi M, Muratori M, Forti G \& Baldi E 1999 Identification and characterization of a novel functional estrogen receptor on human sperm membrane that interferes with progesterone effects. Journal of Clinical Endocrinology and Metabolism 84 1670-1678.

Marchetti B, Serra PA, L'Episcopo F, Tirolo C, Caniglia S, Testa N, Cioni S, Gennuso F, Rocchitta G, Desole MS et al. 2005 Hormones are key actors in gene $\mathrm{x}$ environment interactions programming the vulnerability to Parkinson's disease: glia as a common final pathway. Annals of the New York Academy of Sciences 1057 296-318.

McCarthy MM 2008 Estradiol and the developing brain. Physiological Reviews 88 91-124.

Miller DB, Ali SF, O'Callaghan JP \& Laws SC 1998 The impact of gender and estrogen on striatal dopaminergic neurotoxicity. Annals of the New York Academy of Sciences 844 153-165.

Milner TA, Lubbers LS, Alves SE \& McEwen BS 2008 Nuclear and extranuclear estrogen binding sites in the rat forebrain and autonomic medullary areas. Endocrinology 149 3306-3312.

Nilsen J \& Brinton RD 2004 Mitochondria as therapeutic targets of estrogen action in the central nervous system. Current Drug Targets. CNS and Neurological Disorders 3 297-313.

Olichon A, Baricault L, Gas N, Guillou E, Valette A, Belenguer P \& Lenaers G 2003 Loss of OPA1 perturbates the mitochondrial inner membrane structure and integrity, leading to cytochrome $c$ release and apoptosis. Journal of Biological Chemistry 278 7743-7746.

Pawlak J, Karolczak M, Krust A, Chambon P \& Beyer C 2005 Estrogen receptor-alpha is associated with the plasma membrane of astrocytes and coupled to the MAP/Src-kinase pathway. Glia 50 270-275.

Pellerin L, Bouzier-Sore AK, Aubert A, Serres S, Merle M, Costalat R \& Magistretti PJ 2007 Activity-dependent regulation of energy metabolism by astrocytes: an update. Glia 55 1251-1262.

Poole AC, Thomas RE, Andrews LA, McBride HM, Whitworth AJ \& Pallanck LJ 2008 The Pink1/Parkin pathway regulates mitochondrial morphology. PNAS 105 1638-1643.

Quadros PS, Pfau JL \& Wagner CK 2007 Distribution of progesterone receptor immunoreactivity in the fetal and neonatal rat forebrain. Journal of Comparative Neurology 504 42-56.

Rojo M, Legros F, Chateau D \& Lombes A 2002 Membrane topology and mitochondrial targeting of mitofusins, ubiquitous mammalian homologs of the transmembrane GTPase Fzo. Journal of Cell Science 115 1663-1674.

Roof RL \& Hall ED 2000 Gender differences in acute CNS trauma and stroke: neuroprotective effects of estrogen and progesterone. Journal of Neurotrauma 17 367-388.

Rune GM, Lohse C, Prange-Kiel J, Fester L \& Frotscher M 2006 Synaptic plasticity in the hippocampus: effects of estrogen from the gonads or hippocampus? Neurochemical Research 31 145-155.

Santagati S, Melcangi RC, Celotti F, Martini L \& Maggi A 1994 Estrogen receptor is expressed in different types of glial cells in culture. Journal of Neurochemistry 63 2058-2064.

Santel A \& Fuller MT 2001 Control of mitochondrial morphology by a human mitofusin. Journal of Cell Science 114 867-874.

Santel A, Frank S, Gaume B, Herrler M, Youle RJ \& Fuller MT 2003 Mitofusin-1 protein is a generally expressed mediator of mitochondrial fusion in mammalian cells. Journal of Cell Science 116 2763-2774.

Schapira AH 2008 Mitochondria in the aetiology and pathogenesis of Parkinson's disease. Lancet Neurology 7 97-109.
Von Schassen C, Fester L, Prange-Kiel J, Lohse C, Huber C, Bottner M \& Rune GM 2006 Oestrogen synthesis in the hippocampus: role in axon outgrowth. Journal of Neuroendocrinology 18 847-856.

Schousboe A, Bak LK, Sickmann HM, Sonnewald U \& Waagepetersen HS 2007 Energy substrates to support glutamatergic and GABAergic synaptic function: role of glycogen, glucose and lactate. Neurotoxicity Research 12 263-268.

Shulman LM 2007 Gender differences in Parkinson's disease. Gender Medicine 4 8-18.

Simpkins JW, Yang SH, Wen Y \& Singh M 2005 Estrogens, progestins, menopause and neurodegeneration: basic and clinical studies. Cellular and Molecular Life Sciences 62 271-280.

Singh M, Dykens JA \& Simpkins JW 2006 Novel mechanisms for estrogen-induced neuroprotection. Experimental Biology and Medicine $231514-521$.

Smirnova E, Griparic L, Shurland DL \& van der Bliek AM 2001 Dynamin-related protein Drp1 is required for mitochondrial division in mammalian cells. Molecular Biology of the Cell 12 2245-2256.

Stein DG, Wright DW \& Kellermann AL 2008 Does progesterone have neuroprotective properties? Annals of Emergency Medicine 51 164-172.

Stirone C, Duckles SP, Krause DN \& Procaccio V 2005 Estrogen increases mitochondrial efficiency and reduces oxidative stress in cerebral blood vessels. Molecular Pharmacology 68 959-965.

Stojanovski D, Koutsopoulos OS, Okamoto K \& Ryan MT 2004 Levels of human Fis 1 at the mitochondrial outer membrane regulate mitochondrial morphology. Journal of Cell Science 117 1201-1210.

Sugioka R, Shimizu S \& Tsujimoto Y 2004 Fzo1, a protein involved in mitochondrial fusion, inhibits apoptosis. Journal of Biological Chemistry 279 52726-52734.

Taguchi N, Ishihara N, Jofuku A, Oka T \& Mihara K 2007 Mitotic phosphorylation of dynamin-related GTPase Drpl participates in mitochondrial fission. Journal of Biological Chemistry 282 11521-11529.

Thomas B \& Beal MF 2007 Parkinson's disease. Human Molecular Genetics 16 R183-R194.

Yaffe MP 1999 The machinery of mitochondrial inheritance and behavior. Science 283 1493-1497.

Yager JD \& Chen JQ 2007 Mitochondrial estrogen receptors - new insights into specific functions. Trends in Endocrinology and Metabolism 18 89-91.

Yang SH, Liu R, Perez EJ, Wen Y, Stevens SM Jr, Valencia T, BrunZinkernagel AM, Prokai L, Will Y, Dykens J et al. 2004 Mitochondrial localization of estrogen receptor beta. PNAS 101 4130-4135.

Yoon Y, Krueger EW, Oswald BJ \& McNiven MA 2003 The mitochondrial protein hFis1 regulates mitochondrial fission in mammalian cells through an interaction with the dynamin-like protein DLP1. Molecular and Cellular Biology 23 5409-5420.

Zheng J \& Ramirez VD 1999 Rapid inhibition of rat brain mitochondrial proton F0F1-ATPase activity by estrogens: comparison with $\mathrm{Na}+, \mathrm{K}+-$ ATPase of porcine cortex. European Journal of Pharmacology 368 95-102.

Zuchner S, Mersiyanova IV, Muglia M, Bissar-Tadmouri N, Rochelle J, Dadali EL, Zappia M, Nelis E, Patitucci A, Senderek J et al. 2004 Mutations in the mitochondrial GTPase mitofusin 2 cause CharcotMarie-Tooth neuropathy type 2A. Nature Genetics 36 449-451.

Received in final form 25 July 2008

Accepted 27 August 2008

Made available online as an Accepted Preprint 27 August 2008 